\title{
Understanding ML driven HPC: Applications and Infrastructure
}

\author{
Geoffrey Fox ${ }^{1}$, Shantenu Jha ${ }^{2,3}$ \\ ${ }^{1}$ Indiana University, Bloomington, IN \\ ${ }^{2}$ Rutgers, State University of New Jersey, Piscataway, NJ 08854, USA \\ ${ }^{3}$ Brookhaven National Laboratory, Upton, New York, 11973
}

\begin{abstract}
We recently outlined the vision of "Learning Everywhere" which captures the possibility and impact of how learning methods and traditional HPC methods can be coupled together. A primary driver of such coupling is the promise that Machine Learning (ML) will give major performance improvements for traditional HPC simulations. Motivated by this potential, the ML around HPC class of integration is of particular significance. In a related follow-up paper, we provided an initial taxonomy for integrating learning around HPC methods. In this paper, which is part of the Learning Everywhere series, we discuss "how" learning methods and HPC simulations are being integrated to enhance effective performance of computations. This paper identifies several modes - substitution, assimilation, and control, in which learning methods integrate with HPC simulations and provide representative applications in each mode. This paper discusses some open research questions and we hope will motivate and clear the ground for MLaroundHPC benchmarks.
\end{abstract}

\section{INTRODUCTION AND MOTIVATION}

The convergence of HPC and learning methodologies provides a promising approach to major performance improvements. Traditional HPC simulations are reaching the limits of original progress. The end of Dennard scaling of transistor power usage, and the end of Moores Law as originally formulated has yielded fundamentally different processor architectures. The architectures continue to evolve, resulting in highly costly, if not damaging churn in scientific codes that need to be finely tuned to extract the last iota of parallelism and performance. This approach to high-performance scientific computing is simply unsustainable.

In domain sciences such as biomolecular sciences, advances in statistical algorithms and runtime systems have enabled extreme scale ensemble based applications [1] to overcome limitations of traditional monolithic simulations. However, in spite of several orders of magnitude improvement in efficiency from these adaptive ensemble algorithms, the complexity of phase space and dynamics for modest physical systems, require additional orders of magnitude improvements and performance gains. Integrating traditional HPC approaches with machine learning methods holds significant promise towards overcoming these barriers.

It has always been necessary to improve the effectiveness of simulations; however, its necessity and significance increases drastically at large-scales. First, there is a need to enhance, if not preserve computational efficiency at scale. Applying highperformance computing capabilities at (exa-)scale, leads to the possibility of greater scientific inefficiency in computational campaigns. For example, greater computational capacity might generate relatively greater correlations and lower sampling, and thus less independent data and exploration. Algorithms, methods and campaign strategies that worked at lower scales are not necessarily suitable at greater scales. Second, traditional computational campaigns have not exploited the intermediate data from high-performance computing to their fullest: computational campaigns have been conducted in a static, if not ad hoc fashion based upon initial assumptions and states. The implications of static computational campaigns will be exacerbated at scale, and thus novel algorithms, methods and campaign strategies are needed that employ sophisticated learning to utilize and adapt to intermediate data products.

In many application domains, the integration of ML into computations is a promising way to obtain large performance gains, and presents an opportunity to jump a generation of simulation enhancements. For example, one can view the use of learned surrogates as a performance boost that can lead to huge speedups, as calculation of a prediction from a trained network can be many orders of magnitude faster than full execution of the simulation [2], [3]. In addition to the use of learning for advanced sampling as illustrated above, simple examples are the use of a surrogate to represent a chemistry potential, or a larger grain size to solve the diffusion equation underlying cellular and tissue level simulations.

This paper explores opportunities at the interface between high-performance simulations and machine learning. Specifically, it investigates how ML driven HPC simulations - which based upon the taxonomy introduced in Ref. [2] is referred to as the "ML around HPC" - can pervasively enhance high-performance computational science. It attempts to answer questions such as: How and where can ML effectively enhance HPC simulations? When should ML methods substitute traditional simulations? Which ML methods are promising? What are the general motifs or patterns of interaction between ML and HPC?

In order to provide a quantitative metric by which to measure and answer some of these questions, it is necessary to distinguish between traditional performance measured by operations per second or benchmark scores, from the effective performance that one gets by combining learning with simulation which gives increased scientific performance - as 
determined by a suitable metric and measure, without changing the traditional system characteristics.

In general, there are three types of performance that require distinction: the first, traditional system or application performance, which is measured by typical scaling, utilization or operations by second and benchmark scores. The second is the improvement in the computational investigation of the scientific process, as measured by time-to-solution (for a given resource amount) or another scientific metric. The third measure of performance, is the increase in either the learning phase due to being trained with (physically meaningfully) simulations, or the improvement in the simulation due to being interaction with learning phase.

In cases where there is a (tight) coupling between the learning and simulation components, the second measure of performance is of paramount importance. It motivates the notion of crossover point defined as the point in configuration space at which the learning method is either more performant - efficient (e.g., same quality of results produced with less computing), or better (e.g., produces better results than possible via first principles / simulations), or faster than simulations (e.g., speeding up or classic effective performance a la reduced order modeling). Crossover points are dependent upon several factors including the complexity of underlying model and problem, availability of surrogate, the sensitivity to the ratio of cost / value of data (e.g, is it better to have lots of cheap and low quality data, or small amount of high quality data).

This paper is a follow on from Learning Everywhere [2] and an accompaniment to the article on "Taxonomy of MLaroundHPC" as part of the IEEE eScience 2019. In Section II, we summarize the high-level organization of MLforHPC, followed by an taxonomy of different MLaroundHPC applications. In Section III, we focus on MLaroundHPC investigating the different modes, mechanisms and functional motivations of integration of ML around HPC. We also discuss some canonical examples of the different modes of integration. We will use insights gained from an investigation of these issues to discuss open issues and research challenges in cyberinfrastructure - algorithms \& methods, software and hardware, that must be addressed in the near and intermediate term.

We thank the organizers of the IEEE eScience 2019 for the opportunity to contribute to the Vision Track. We believe eScience conference series has an important and distinguished track record of bringing the data sciences - methods and infrastructure, closer to traditional simulation based science. We hope this article will help the eScience Steering Committee to keep the conference series aligned with the thinking, needs and future directions of the community to push the boundaries of computational and data driven discovery.

\section{LEARNING EVERYWHERE}

We have identified [2] several important distinctly different links between machine learning (ML) and HPC. We term the full area MLandHPC and define two broad categories [4]: HPCforML and MLforHPC. HPCforML uses HPC to execute and enhance ML performance, or using HPC simulations to train ML algorithms (theory guided machine learning), which are then used to understand experimental data or simulations. On the other hand MLforHPC uses ML to enhance HPC applications and systems, where big data comes from the computation and/or experimental sources that are coupled to ML and/or HPC elements. MLforHPC can be further subdivided as MLaroundHPC, MLControl, MLAutotuningHPC, and MLafterHPC described in detail below.

The MLforHPC category covers all aspects of machine learning interacting with computation typically implemented as HPC. The sub-categories are useful but incomplete, and definitely not always precise. There is a need to improve the conceptual understanding of the different facets and dimensions of MLforHPC. We delineate the initial types of MLforHPC we have identified:

\section{A. MLaroundHPC}

Using ML to learn from simulations and produce learned surrogates for the simulations. This increases effective performance for strong scaling where we keep the problem fixed but run it faster or run the simulation for a longer time interval such as that relevant for biological systems. It includes SimulationTrainedML where the simulations are performed to directly train an AI system rather than the AI system being added to learn a simulation. Some common ways in which MLaroundHPC is used, include:

1) MLaroundHPC: Learning Outputs from Inputs Simulations performed to directly train an AI system, rather than AI system being added to learn a simulation [5], [6].

2) MLaroundHPC: Learning Simulation Behavior ML learns behavior replacing detailed computations by ML surrogates [7], [8]

3) MLaroundHPC: Faster and Accurate PDE Solutions Efficient numerical solution of PDEs is one of the most costly computations in many simulations, and solving high dimensional PDEs such as the diffusion equation has been notoriously difficult. Recent ML accelerated algorithms [8] for solving high-dimensional nonlinear PDEs are effective for a wide variety of problems, in terms of both accuracy and speed. These algorithms [9] approximate the solution highdimensional PDEs such as the diffusion equation using a "Deep Galerkin Method (DGM)", and train their network on batches of randomly sampled time and space points. These new AI accelerated approaches [10], [11] open up a host of possibilities in materials, physics and cosmology, and scientific computing more generally.

4) MLaroundHPC: New Approach to Multi-Scale Modeling Effective potential is an analytic, quasi-emperical or quasi-phenomological potential that combines multiple, perhaps opposing, effects into a single potential. For example, we have a model specified at a microscopic scale and we define a coarse graining to a different scale with macroscopic entities defined to interact with effective dynamics specified in some fashion such as an effective potential or effective interaction graph. Machine learning is ideally suited for defining 
effective potentials and order parameter dynamics, and shows significant promise to deliver orders of magnitude performance increases over traditional coarse-graining and order parameter approaches. See well established methods [12]-[17]

\section{B. MLControl}

Two representative scenarios are:

1) Experiment Control Using simulations (possibly with HPC) in control of experiments and in objective driven computational campaigns [18]. Here the simulation surrogates are very valuable to allow real-time predictions. Examples about: Material Science [19]-[21], Fusion [22], Nano [23]

2) Experiment Design A big challenge is the uncertainty in the precise model structures and parameters. Model-based design of experiments (MBDOE) assists in the planning of highly effective and efficient experiments - it capitalizes on the uncertainty in the models to investigate how to perturb the real system to maximize the information obtained from experiments. MBDOE with new ML assistance [24] identifies the optimal conditions for stimuli and measurements that yield the most information about the system given practical limitations on realistic experiments.

\section{MLAutoTuning}

Captures the scenario where ML is used to efficiently configure the HPC computations. MLAutoTuning can be applied at multiple distinct points, and can be used for a range of tuning and optimization objectives. For example: (i) mix of performance and quality of results using parameters provided by learning network [4], [25]-[28]; (ii) choose the best set of "computation defining parameters" to achieve some goal such as providing the most efficient training set with defining parameters spread well over the relevant phase space [29], [30]; (iii) tuning model parameters to optimize model outputs to available empirical data [31]-[34].

\section{MLafterHPC}

ML analyzing results of HPC as in trajectory analysis and structure identification in biomolecular simulations [35].

\section{MLAROUNDHPC ClassifiCATION AND EXEMPLARS}

The interaction between models and simulation data occurs in two directions: (i) The problem of how to use multimodal data to inform complex models in the presence of uncertainty, and (ii) How, where, when, and from which source to acquire simulation data to optimally inform models with respect to a particular goal or goals is fundamentally an optimal experimental design problem. Creating the conceptual and technological framework in which models optimally learn from data and data acquisition is optimally guided by models presents significant challenges systems of interest are complex, multiscale, strongly interacting/correlated, and uncertain.

It is important to separate the modes and mechanics of how learning is integrated with HPC simulations, from the functional motivations of doing so. Based upon an extensive analysis of the current state of the field, the three primary modes and mechanisms for integrating learning with HPC simulations are - substitution, assimilation and control. Each represents a broad range of subcategories and possibilities, which no doubt will change rapidly as the state of theory and practice evolves.

Independent of the modes and mechanisms of integration, we find that there are three functional drivers of the integration:

1) Improving Simulations: The essence of this driver is to use learning to configure and select simulations effectively. There are several approaches to learning the configuration of physical system being studied, ranging from improving the learning models using simulation data dynamically, to using models to determine simulation configurations and/or parameters, as well as possibly learn configurations of system and software for improved performance on particular hardware and input parameters [1].

2) Learn Structure, Theory and Model for Simulation: Here the simulations are used to gradually improve the model or theory, which are in turn used to improve simulations in some fashion (e.g., as per previous point). As simulations proceed the model learns the structure or even underlying principles, and is gradually refined either by coarse-graining or using better approximations to the effective potentials [36].

3) Learn to make Surrogates: An increasingly common and important driver is the use of ML (which are often deep networks) to learn the function representing the output of the simulation. Such learned representations also often referred to as surrogates, can be used to determine either the parameters or the effective "fields" [7], [8].

It is no surprise that the first driver is the most widely investigated and applied; the rate of progress in the second and third drivers is rapid and impressive. We now discuss the primary modes and mechanisms in which the above three scenarios are often implemented.

\section{A. Substitution}

In this mode, a surrogate model is used to substitute an essential element of the original simulation (method). The surrogate model is used to create multi-scale or coarse grained surrogate modeling, which could either learn the structure or theory of original simulation.

Example: Roitberg et al. [15] trained a network on using fine grained Quantum Mechanical DFT calculations. The resulting ANI-1 model was shown to be chemically accurate, transferrable, with a performance similar to a classical force field, thus enabling ab-initio molecular dynamics at a fraction of the cost of "true" DFT ab-initio simulations. Extensions of their work with an active learning (AL) approach demonstrated that proteins in an explicit water environment can be simulated with a NN potential at DFT accuracy [17].

In general the focus has been on achieving DFT-level accuracy because NN potentials are not cheaper to evaluate than most classical empirical potentials. However, replacing solvent-solvent and solvent-solute interactions, which typically make up $80 \%-90 \%$ of the computational effort in a classical all-atom, explicit solvent simulation, with a NN potential 
promises large performance gains at a fraction of the cost of traditional implicit solvent models and with an accuracy comparable to the explicit simulations [37].

\section{B. Assimilation}

In this mode, data from simulations, offline external constraints, or real-time experiments are integrated into physicsbased models, which are then assimilated into traditional simulations. The canonical examples are improving the Hamiltonian or Force Fields, or in classical data assimilation studies such as in climate and weather prediction, where in data assimilation involves continuous integration of time dependent simulations with observations to correct the model, which are combined and updated with traditional simulation model.

Example: Current climate models are too coarse to resolve many of the atmospheres most important processes. Traditionally, these subgrid processes are heuristically approximated in so-called parameterizations. However, imperfections in these parameterizations, especially for clouds, have impeded progress toward more accurate climate predictions for decades. Cloud resolving models alleviate many of the gravest issues of their coarse counterparts but will remain too computationally demanding for climate change predictions for the foreseeable future. In Ref. [38], a deep neural network is trained to represent all atmospheric subgrid processes in a climate model by learning from a multiscale model in which convection is treated explicitly. The trained neural network then replaces the traditional subgrid parameterizations in a global general circulation model in which it freely interacts with the resolved dynamics and the surface-flux scheme. The prognostic multiyear simulations are stable and closely reproduce not only the mean climate of the cloud-resolving simulation but also key aspects of variability, including precipitation extremes and the equatorial wave spectrum. Furthermore, the neural network approximately conserves energy despite not being explicitly instructed to. Ref. [38] uses deep learning to leverage the power of short-term cloud-resolving simulations for climate modeling; the approach is fast and accurate, thereby showing the potential of machine-learningbased approaches to climate model development.

\section{Control and Adaptive Execution}

In this mode, the simulation (or ensemble of simulations) are controlled towards important and interesting parts of simulation phase space. Sometimes this involves determining the parameters of the next stage (iteration) of simulations based upon intermediate data. Sometimes the entire campaign can be adaptively steered towards an objective, which in turn could involve getting better data via active learning based upon an objective function, or use a policy-based reinforcement learning approach to steer the computational campaign.

Example: A fundamental problem that currently pervades diverse areas of science and engineering is the need to design expensive computational campaigns (experiments) that are robust in the presence of substantial uncertainty. A particular interest lies in effectively achieving specific objectives for systems that cannot be completely identified. For example, there may be big data but the data size may still pale in comparison with the complexity of the system, or the available data may be scarce due to the prohibitive cost of experiments.

A framework for the objective driven experiment design (ODED) will support the integration of scientific prior knowledge on the system with data generated via simulations, quantify the uncertainty relative to the objective, and design optimal experiments that can reduce the uncertainty and thereby directly contribute to the attainment of the objective.

\section{MLAROUNDHPC CYBERINFRASTRUCTURE}

We distill the analysis and description of MLAroundHPC modes and examples into three cyberinfrastructure categories: (i) algorthms, benchmarks and methods; (ii) system software and runtime, and (iii) hardware.

\section{A. Algorithms, Benchmarks and Methods}

The methodologies by which experiments inform theory, and theory guides experiments, remain ad hoc, particularly when the physical systems under study are multiscale, largescale, and complex. Off-the-shelf machine learning methods are not the answer; these methods have been successful in problems for which massive amounts of data are available and for which a predictive capability does not rely upon the constraints of physical laws. The need to address this fundamental problem has become urgent, as computational campaigns at pre-exascale, and soon exascale, will entail models that span wider ranges of scales and represent richer interacting physics. Open issues and research questions include:

1) Does the crossover point - at which prediction based approaches are better than traditional HPC simulations, suggest or motivate a need to redesign some simulation algorithms so that MLforHPC effective? Similarly, if HPC simulations are going to serve as important sources of data generation, is there an opportunity to devise novel learning algorithms and methods so as to support more effective MLforHPC?

2) Simulations are simply 4D time-series data! Thus, there ought to be important analogies between time series ML research and MLforHPC.

3) Importance of canonical problems: Understanding of which learning methods work, why and for which problems. How do we develop benchmarks to highlight different application and system features? By extension, how do we develop proxy apps to represent the applications?

4) Understanding Performance: What are the performance metrics that represent the integrated working of learning and simulations? What is the comparison in scientific discovery between the large increase in performance possible with true exascale machines and the exascale (or zettascale) effective performance possible with MLforHPC? How does the interplay of raw performance and effective performance influence the mapping of applications to compute systems? 


\section{B. System Software and ML-HPC Runtime Systems:}

MLforHPC needs to support large scale simulations and learning, and their integrated and concurrent execution. The combined workload - distinct ML and HPC computation tasks, will need to be run flexibly. For example, sometime the HPC simulation will be used to generate training data and then run ML; sometimes the ML will be responsible for inference as HPC simulations are generating data. On occasions, HPC simulations will run after Learning (or vice versa), but sometimes they will be intertwined in a single job. Thus, it is imperative to understand the general control and coupling between Learning elements (L), HPC Simulation (S). In many cases a third general component - experiments or observations (E) may also be needed.

There are several dimensions to characterize the coupling between these components, including temporal and data volumes. The former will determine the type of algorithms and learning approaches taken; the latter software scaling and performance requirements. Furthermore, the specific type of coupling could yield steering or control. (Component $\mathrm{X}$ is said to steer component $\mathrm{Y}$, when $\mathrm{X}$ provides the relevant information to determine the execution of $\mathrm{Y}$. Whether $\mathrm{Y}$ accepts or not, is determined by additional considerations such as objective, policy, etc. Steering is a necessary condition for control; not all steering represents control).

Different scenarios for coupling information and control flow, between different elements E, L and S. Scenario III covers two possibilities: learning element controls experimental data source, or Simulation controls experimental data source. The logical coupling disregards the physical location of the elements, e.g., E could be on an Edge device or a HPC cluster.

In order to support the real-time application requirements, it is important to achieve near real-time training and prediction to control or steer S or E. For example, build low dimensional representation of states from trajectory analysis. The strong scaling of just $\mathrm{L}$ is inadequate, and scaling properties of integrated $\mathrm{L}+\mathrm{S}$ elements are needed for MLaroundHPC applications. A preliminary analysis suggests that this can be achieved by adapting the ratio of the cardinality of $\mathrm{L}, \mathrm{S}$ and $\mathrm{E}$, viz., $\mathrm{N}_{L}$ (the number of learning) to $\mathrm{N}_{S}$ (number of simulation elements) being time-dependent. These translate into support for coordinated execution of a large number of concurrent and heterogeneous simulations as well as enabling adaptive execution and resource partitioning between simulation and learning elements.

Additional considerations that a runtime system to support the concurrent execution of ML and HPC elements include: Is a single run-time system possible that will be able to support the different classes of MLforHPC, varied data rates (from trivial to $\mathrm{O}(100) \mathrm{GB} / \mathrm{s}$ ) and latency tolerance (from $<\mathrm{O}(1) \mathrm{s}$ to $\mathrm{O}(100)$ s)? Can a single runtime system support the full range of fine-grained to coarse-grained coupling between learning and simulation components? What are the considerations and constraints that inform performance guarantees and workload balancing (e.g., dynamically varying the number of learning elements and simulation elements)?

\section{Hardware and Platform Configuration}

What fraction of time (resource) is spent in ML component and how does this change with scale? What is the frequency and extent of coupling between learning and simulations? Insight into the above questions could influence optimal architecture, e.g., when the ratio of learning (training and inference) is small, a classic supercomputing architecture linked to a separate learning system might be acceptable if not optimal. Conversely, when the ratio is large, a tightly integrated system supercomputer might be more suitable? What are the quantitative determinants of an optimal platform? How should a balanced system across a range of MLaroundHPC applications be designed: fixed dollars for learning vs simulations, or a dollar distribution that tracks the relative computing intensity? Or one that optimizes inference phase versus training phase? Should future HPC platforms be designed to support both phases, or is platform specialization for training and inference most effective?

Hardware and platform considerations that arise from uncertainty in technology roadmap and pricing include:

1) Role and importance of heterogeneous accelerators, especially as a new generation of ML accelerators is developed that may not be in simulations (currently GPU accelerators often useful in both ML and simulation); (ii) As we expect time series in data assimilation likely to use RNNs and the importance and pervasiveness of RNN to increase, when should Recurrent neural networks RNN (commonly used in learning sequences) need different accelerators from convolutional neural nets)?

2) Requirements also suggest the need for fast I/O and internode communication to enable ML and Computation to run together and exchange information with each other and with sources of streaming data. It is not evident how large and fast disks should be organized, but disks on each node seem required to hold data to be exchanged between simulation and ML components of a job and for accumulating training data and NN weights.

3) Need ML optimization and Simulation optimization spread through machine and fast ways for ML and simulation to exchange data. Given the emergence of cloudlets (aka fog computing), there is a need to support HPC/Cloud, Fog and Edge platforms, as well as their integration.

\section{Discussion AND CONCLUSION}

The state of HPC in 2020 presents challenges and opportunities. On the one hand, HPC methods and platforms are becoming pervasive and necessary for scientific advances. On the other, traditional HPC computations are reaching various limits. The implications of hardware and architectural trends are well known: the end of Dennard scaling and of Moore's Law as originally formulated, is yielding very different processor architectures; achieving performance gains is becoming harder, while requiring significant, if not unsustainable software investment and algorithmic reformulation. 
The HPC community has - somewhat naively, assumed that as long as performance gains from hardware are possible, traditional simulation based methods will continue to provide increased scientific insight. However, without careful examination of the scientific efficiency or effective performance of existing simulation and first principles methods, it is not obvious that traditional simulations represent the optimal approach at exascale and beyond, and on subsequent generation of supercomputers. In other words, we may be reaching limits of both hardware and methodological performance gains.

There is a need for major functionality and performance increases that are independent of changes in hardware. In traditional HPC the prevailing orthodoxy "Faster is Better" or what is worse, the conflation of "bigger" with "better" has driven the quest for hierarchical parallelism to speeding up single units of works. Relinquishing the orthodoxy of hierarchical parallelism as the primary route to performance is necessary. In fact, there is a need to carefully reconsider discredited approaches, while adopting the new paradigms.

Enter "Learning Everywhere" - the essential idea of which, is that by embedding learning methods and approaches in all aspects of the system configuration and application execution, the effective performance can be dramatically improved.

There is a regime where learning based predictive approaches are going to outperform first-principles and simulation-based approaches. The exact sweet spot or crossover point is non-trivial to determine: it will be application specific, depend upon complexity of learned models, volume and cost of data, as well as effectiveness and cost of simulations, inter alia. However, the underlying idea that surrogate learned models will represent effective performance improvements over traditional approaches, is a powerful one, and is an important generalization of the multi-scale, coarsegrained approaches used in many physical sciences.

Learning Everywhere is one specific example of the paradigmatic shift in scientific computing that will be needed at extremes scales. Statistical computing, which incorporates elements of approximate computing, uncertainty minimization and other objective driven dynamic computational campaigns will substitute predefined "static" computational campaigns. Nowhere is the impact of this likely to be greater than in those domains which require the assimilation of streaming and dynamic data, or computational campaigns that are statistical in nature and driven towards optimality or objectives. These methodological innovations will heighten the importance of adaptive execution of ensembles of heterogeneous models, and will require novel scalable middleware systems.

Looking Ahead: The pace of innovation in learning for science is intense and rapid. No surprises it is difficult to predict the exact trajectory or state for anything but the immediate future. It is safe to expect major impact of ML on science in essentially all areas and in multiple modes: many traditional physics applications including simulations and Monte Carlo methods are being reformulated using learning approaches [39].

Integrating learning with HPC provides an opportunity to enhance methods and for some domains such as molecular science to jump ahead. For other field, such as high-energy physics, that did not invest and anticipate the disruptions arising from end of Dennard and Moore's law resulting in the explosion of heterogeneous computing and accelerators, it presents an important opportunity to simply by-pass and leapfrog a generation of simulation enhancements!

Impressive, if not inspiring papers that apply learning to societally important problems such as climate change [40] are valuable harbingers. Molecular sciences has been an enthusiastic adopter of learning methods: Machine Learning used in materials simulation to aid the design of new materials and to understand properties [41]; predict reaction coordinates [42]; and enhanced sampling [43] and dynamics on long timescales [44]. Even as the use of ML in science changes, important advances in the way ML is formulated are happening. For example, Ref. [45] shows how to scale CNNs as problems scale - which will be crucial in using NN for complex physics systems. In fact, Ref. [46] uses ODEs to build a continuous neural network rather than one built from a set of layers.

Enhancements to ML will be necessary to deliver on new and promising uses of learning in science, such as the application of DL for time series - geospatial and simulation trajecctory data (which are simply 4D time series). These problems can be formulated as graphs spatially (with convolutional NNs) and as sequences (with recurrent NNs) in time. Many HPC Cloud-Edge systems will provide such time series, and also reinforce the need for real-time response which raises difficult trade-offs between performance and functionality and highlights the role of HPC 47]. In general, the pace of methodological innovation and application requirements will have important implications for the cyberinfrastructure developed and deployed for the science of tomorrow.

Acknowledgement This work was partially supported by NSF CIF21 DIBBS 1443054 and nanoBIO 1720625. SJ was partially supported by ExaLearn - a DOE Exascale Computing project.

\section{REFERENCES}

[1] Peter M Kasson and Shantenu Jha. Adaptive ensemble simulations of biomolecules. Current Opinion in Structural Biology, 52:87 - 94, 2018. Cryo electron microscopy: the impact of the cryo-EM revolution in biology Biophysical and computational methods - Part A.

[2] Geoffrey C. Fox, James A. Glazier, J. C. S. Kadupitiya, Vikram Jadhao, Minje Kim, Judy Qiu, James P. Sluka, Endre Somogy, Madhav Marathe, Abhijin Adiga, Jiangzhuo Chen, Oliver Beckstein, and Shantenu Jha. Learning everywhere: Pervasive machine learning for effective highperformance computation. In IEEE International Parallel and Distributed Processing Symposium Workshops, IPDPSW 2019, Rio de Janeiro, Brazil, May 20-24, 2019, pages 422-429, 2019.

[3] João Marcelo Lamim Ribeiro and Pratyush Tiwary. Toward achieving efficient and accurate Ligand-Protein unbinding with deep learning and molecular dynamics through RAVE. Journal of chemical theory and computation, 15(1):708-719, 8 January 2019.

[4] Jeff Dean. Machine learning for systems and systems for machine learning. In Presentation at 2017 Conference on Neural Information Processing Systems, 2017.

[5] Frank Noé, Simon Olsson, Jonas Köhler, and Hao Wu. Boltzmann generators - sampling equilibrium states of Many-Body systems with deep learning. 4 December 2018.

[6] Katsuhiro Endo, Katsufumi Tomobe, and Kenji Yasuoka. Multi-step time series generator for molecular dynamics. In Thirty-Second AAAI Conference on Artificial Intelligence. aaai.org, 2018. 
[7] Wolfgang Gentzsch. Deep learning for fluid flow prediction in the cloud.

[29] Valeriy Gavrishchaka, Olga Senyukova, and Mark Koepke. Synergy of https:/www linkedin com/pulse/deep-learning-fluid-flow-prediction-cloud-wolfgaploy gresthosed reasoning and machine learning in biomedical applicaDecember 2018. Accessed: 2019-3-1.

[8] Jiequn Han, Arnulf Jentzen, and Weinan E. Solving high-dimensional partial differential equations using deep learning. Proceedings of the National Academy of Sciences of the United States of America, 115(34):8505-8510, 21 August 2018.

[9] Justin Sirignano and Konstantinos Spiliopoulos. DGM: A deep learning algorithm for solving partial differential equations. Journal of computational physics, 375:1339-1364, 15 December 2018.

[10] Rohit K Tripathy and Ilias Bilionis. Deep UQ: Learning deep neural network surrogate models for high dimensional uncertainty quantification. Journal of computational physics, 375:565-588, 15 December 2018.

[11] Maziar Raissi, Paris Perdikaris, and George Em Karniadakis. Physics informed deep learning (part II): Data-driven discovery of nonlinear partial differential equations. 28 November 2017.

[12] Jrg Behler and Michele Parrinello. Generalized Neural-Network Representation of High-Dimensional Potential-Energy Surfaces. Physical Review Letters, 98(14):146401, April 2007.

[13] Jrg Behler. First Principles Neural Network Potentials for Reactive Simulations of Large Molecular and Condensed Systems. Angewandte Chemie International Edition, 56(42):12828-12840, 2017.

[14] Keith T. Butler, Daniel W. Davies, Hugh Cartwright, Olexandr Isayev, and Aron Walsh. Machine learning for molecular and materials science. Nature, 559(7715):547, July 2018.

[15] J. S.Smith, O. Isayev, and A. E.Roitberg. ANI-1: an extensible neural network potential with DFT accuracy at force field computational cost. Chemical Science, 8(4):3192-3203, 2017.

[16] Justin S Smith, Benjamin T. Nebgen, Roman Zubatyuk, Nicholas Lubbers, Christian Devereux, Kipton Barros, Sergei Tretiak, Olexandr Isayev, and Adrian Roitberg. Outsmarting Quantum Chemistry Through Transfer Learning. chemrxiv, doi: 10.26434/chemrxiv.6744440.v1, July 2018.

[17] Justin S. Smith, Ben Nebgen, Nicholas Lubbers, Olexandr Isayev, and Adrian E. Roitberg. Less is more: Sampling chemical space with active learning. The Journal of Chemical Physics, 148(24):241733, May 2018.

[18] Francis J. Alexander, Shantenu Jha. Objective driven computational experiment design: An ExaLearn perspective. In Terry Moore, Geoffrey Fox, editor, Online Resource for Big Data and Extreme-Scale Computing Workshop, November 2018.

[19] Kevin Yager. Autonomous experimentation as a paradigm for materials discovery. In Geoffrey Fox Terry Moore, editor, Online Resource for Big Data and Extreme-Scale Computing Workshop, November 2018.

[20] Fang Ren, Logan Ward, Travis Williams, Kevin J Laws, Christopher Wolverton, and Apurva Mehta. Accelerated discovery of metallic glasses through iteration of machine learning and high-throughput experiments. Science advances, 4(4):eaaq1566, April 2018.

[21] Logan Ward. Deep learning, HPC, and data for materials design. In Terry Moore, Geoffrey Fox, editor, Online Resource for Big Data and Extreme-Scale Computing Workshop, November 2018.

[22] Bill Tang. New models for integrated inquiry: Fusion energy exemplar. In Geoffrey Fox Terry Moore, editor, Online Resource for Big Data and Extreme-Scale Computing Workshop, November 2018.

[23] Prasanna V Balachandran, Dezhen Xue, James Theiler, John Hogden, and Turab Lookman. Adaptive strategies for materials design using uncertainties. Scientific reports, 6:19660, 2016.

[24] Jasper Snoek, Hugo Larochelle, and Ryan P Adams. Practical bayesian optimization of machine learning algorithms. In Advances in Neural Information Processing Systems, pages 2951-2959, 2012.

[25] Microsoft Research. AI for database and data analytic systems at microsoft faculty summit. https://youtu.be/Tk16ERLWAbA 2018. Accessed: 2019-1-29.

[26] Tim Kraska, Alex Beutel, Ed H Chi, Jeffrey Dean, and Neoklis Polyzotis. The case for learned index structures. In Proceedings of the 2018 International Conference on Management of Data, SIGMOD '18, pages 489-504, New York, NY, USA, 2018. ACM.

[27] JCS Kadupitiya, Geoffrey C. Fox, Vikram Jadhao. Machine learning for parameter auto-tuning in molecular dynamics simulations: Efficient dynamics of ions near polarizable nanoparticles. Technical report, Indiana University, November 2018.

[28] Venkatesh Botu and Rampi Ramprasad. Adaptive machine learning framework to accelerate ab initio molecular dynamics. International Journal of Quantum Chemistry, 115(16):1074-1083, 2015. 